\title{
CONSERVATIVE MANAGEMENT OF INTRACANAL SEPARATED ENDODONTIC INSTRUMENTS - TREATMENT DECISIONS AND RELATED FACTORS
}

\author{
Tsvetelina Borisova-Papancheva ${ }^{1}$, Silvia Stankova ${ }^{1}$, Slavena Georgieva ${ }^{2}$ \\ ${ }^{1}$ Department of Conservative Dentistry and Oral Pathology, Faculty of Dental Medicine, \\ Medical University of Varna \\ ${ }^{2}$ Dentist, Varna
}

\begin{abstract}
INTRODUCTION: Intracanal separation of endodontic instruments is an adverse event that may not only hinder the endodontic treatment, but may also have an influence on the long-term prognosis and longevity of the tooth.

MATERIALS AND METHODS: After studying a series of related articles and publications we have reviewed and compared the different conservative treatment options for the management of intracanal fractured instruments.

RESULTS: Conservative management methods are leaving the fragment in situ and filling the coronal fragment of the root canal, bypassing the fractured instrument or completely removing it from the root canal. Each of these options has its advantages, disadvantages, special indications and every treatment decision is accompanied by possible risks and difficulties. Each case is different and the prognosis depends mainly on the location of the fragment, the root canal system (RCS) anatomy and morphology and, of course, on making the right treatment decision for managing the accident.

CONCLUSION: Separation of endodontic instruments in the root canal (RC) does not necessarily lead to endodontic failure and even in cases when the instrument cannot be removed choosing the adequate treatment procedure can strongly benefit the long-term prognosis of the tooth involved.
\end{abstract}

Keywords: endodontic mishap, separated instrument, bypassing, removal

Address for correspondence:

Tsvetelina Borisova-Papancheva

Faculty of Dental Medicine

Medical University of Varna

84 Tsar Osvoboditel Blvd

Varna

e-mail:dr_borisova@abv.bg

Received: June 14, 2017

Accepted: June 28, 2017

\section{INTRODUCTION}

The potential for instrument separation is present during every standard endodontic treatment. This complication can have an enormous impact on the outcome of the treatment, can be an obstacle for the proper cleaning, shaping and definitive obturation of the RCS and can therefore be the reason for a future endodontic failure (1). Conservative management of intracanal fractured instruments includes 
procedures such as leaving the fragment in situ and obturation of the RCS to the coronal level of the instrument, bypassing the separated fragment or complete removal of the instrument $(2,3)$.

Each of the mentioned above treatment options has its indications, advantages, disadvantages, can lead to possible risks and should be considered and performed after careful evaluation of the exact clinical situation and the related factors.

\section{MATERIALS AND METHODS}

We studied a series of related articles and publications and compared data from numerous researches in order to present and compare the advantages, possible risks, difficulties and consequences of leaving versus completely removing broken instruments from the RC.

Results

Instrument separation can be the result of : (4)

$\diamond$ Improper use of the instrument (5);

$\diamond$ Inadequate access;

$\diamond$ Unusual and unexpected RCS anatomy;

$\diamond$ Manufacturing defects $(4,6)$;

$\diamond$ Rush to use rotary instruments without creating a proper straight-line access and glide path (6)

$\diamond$ Higher breakage rate of rotary files is proved in cases when the root canal was not previously prepared with hand files $(7,8)$;

$\diamond$ Not following the rule that files have to advance slow in the root canal, until resistance is felt (9);

$\diamond$ Not creating glide path to the working length using \#10 and \#15 files (9);

$\diamond$ Overuse of endodontic instruments (9).

The separation of endodontic files happens due to two different fracture mechanisms, acting alone or in combination - tensional stress and cyclic fatigue. The twisting of a file at one end, while it is fixed at the other end, leads to torsional stress. This can happen in straight or curved root canals, in cases when the friction between the file and the root canal wall is sufficient enough to lead to such consequences. Cyclic fatigue results in separation of the file when the cycles of tension and compression are sufficient to cause structural fracture of the file. In most clinical cases both mechanisms work together (10).
When a complication such as intracanal separation of endodontic instrument occurs, a treatment decision should be considered after evaluating factors such as:

$\diamond$ Root canal system anatomy incl. diameter, length, curvature of the root canal, root canal morphology and dentin thickness (3,11-15): In general, curved canals present a higher risk for iatrogenic events than straight root canals. The higher the angle of root curvature is, the more difficult it gets to create a platform around the coronal part of the separated fragment, because of the insufficient dentin thickness (3).

$\diamond$ The stage of root canal preparation $(2,3,15)$ is in correlation with the stage of disinfection of the root canal system. If the separation happens early in the endodontic treatment the root canal is less likely to be properly disinfected than if it happens at the end of the root canal preparation. However, some researches prove that the stage of root canal preparation does not make a difference to the long-term prognosis of the tooth (3).

$\diamond$ Potential complications and iatrogenic events such as ledge formations, perforations incl. stripping perforations, secondary separation of the instruments, used for the removal (2).

$\diamond$ Armamentarium available, such as, for example, operating microscope, ultrasonic tips, etc. $(2,3,11,15)$.

$\diamond$ Presence or absence or periapical pathology $(2,15)$ : In cases without periapical pathology removal may not be necessary and the management could consist in retention or bypassing of the fragment. In cases with apical periodontitis the tooth survival prognosis decreases, so greater efforts should be made towards removal or bypassing of the fractured instrument (3).

$\diamond$ Periodontal and restorative status of the tooth $(3,16)$ : As expected, periodontally compromised teeth or cases of non-restorable teeth do not make good candidates for fragment removal (3).

$\diamond$ Exact location of the fragment in the RC fragments in straight root canals or only partially located in the root curvature are easier to remove $(1,8,9,11-15,17,18)$. Separated instruments, 
Tsvetelina Borisova-Papancheva, Silvia Stankova, Slavena Georgieva

localized in the apical $1 / 3$ of curved root canals are often considered impossible to remove because of the poor visibility and access and are further associated with more possible risks $(19,20)$. It is considered that if $1 / 3$ of the overall length of the fragment is exposed, it can be removed from the RC (11).

$\diamond$ Length of the fragment: Some studies prove that longer fragments are easier to remove than shorter ones. The explanation for this at first unbelievable statement is that with longer fragments it is more likely to create space coronally to allow loosening of the fragment $(17,18,20)$. Other studies prove that the length of the separated fragment is not a significant factor for the treatment decision and the tooth prognosis (13). On the other hand, longer fragments lead to more excessive dentin removal while trying to "loosen" or bypass the fragment, and therefore - a greater chance for iatrogenic events.

$\diamond$ Type of fractured instrument: Lentulo spirals and reamers have proved to be easier to remove according to most studies (17). But more recent researches show that other factors such as RCS anatomy and exact localization of the fragment have much more importance on the outcome than the type of instrument separated $(20,21)$.

$\diamond$ Material of the instrument - stainless steel fragments are considered easier to remove than $\mathrm{Ni}$ Ti instruments, because the latter tend to fracture again $(3,11)$.

$\diamond$ Patient factors such as time constrictions and motivation (15)

$\diamond$ Strategic importance of the tooth involved (15).

Leaving the fragment in situ and filling the root canal to the coronal level of the fragment was a treatment option, recommended before the wide use of an operating microscope and specialized ultrasonic tips (3). Although some studies proved that the broken instrument did not affect the tooth prognosis, this method presents some disadvantages such as (3):

$\diamond$ Compromise of the mechanical and chemical cleansing of the RC;

$\diamond$ Compromise of the proper working length control;

$\diamond$ Compromise of the root canal filling; $\diamond$ Can be a source of anxiety for the patient, who has to further "invest" in an already compromised tooth.

Leaving the fragment is considered applicable if the separation happens at the final stages of the mechanical preparation and chemical irrigation and in cases when the fragment is located in the apical $1 / 3$, beyond the root canal curvature $(2,5)$. It is suggested for teeth treated this way to be filled with thermo plasticized obturation techniques in order to better seal the gap between the fractured instrument and the root canal wall, because of the better flow than other techniques like, for example, cold lateral compaction (5).

Patients should be called for clinical and radiographic follow-ups (2).

Complete removal of the separated instrument describes procedures of creating a gap between the fragment and the root canal wall, leading to loosening of the impacted fragment and final removal. There are different techniques which can result in removing the fragment from the RC.

\section{Indications:}

$\diamond$ When $1 / 3$ or more of the fragment is exposed (11);

$\diamond$ In cases when good visualization is achievable $(11,22)$;

$\diamond$ For fragments located before the root canal curvature (11);

$\diamond$ For fragments located in the coronal $1 / 3$ of the $\mathrm{RC}(11,19,22)$

$\diamond$ For fragments located in straight canals (11). Advantages (3):

$\diamond$ Improves working length control;

$\diamond$ Makes mechanical preparation and chemical cleansing of the RCS possible;

$\diamond$ Makes proper obturation of the RCS achievable. Risks and disadvantages (11):

$\diamond$ Ledge formation;

$\diamond$ Additional preparation and excessive dentin loss are required $(3,22)$;

$\diamond$ Possible stripping perforations (19);

$\diamond$ Possible extrusion of the separated fragment apically or through the apical foramen (19);

$\diamond$ Can result in weakening the root structure and vertical root fractures $(19,22)$; 
$\diamond$ The files, used to remove the fragment, can separate themselves.

\section{Difficulties (11,22):}

$\diamond$ Creating straight access to the fragment in order to achieve proper visibility;

$\diamond$ Not suitable for separated instruments apical to the curvature of the RC;

$\diamond$ Not suitable for fragments in the apical $1 / 3$ of the RC (19).

\section{Techniques for removal:}

$\diamond$ Use of chemical solvents - use of EDTA to soften the root-canal-wall dentin around the fractured instrument (2).

Use of mini forceps, for example Endo Forceps (Roydent, Johnson City, TN) (Fig.1), in cases when there is enough space in the $\mathrm{RC}$ and the fragment is in the coronal $1 / 3$ or extends into the pulp chamber $(2,3)$.

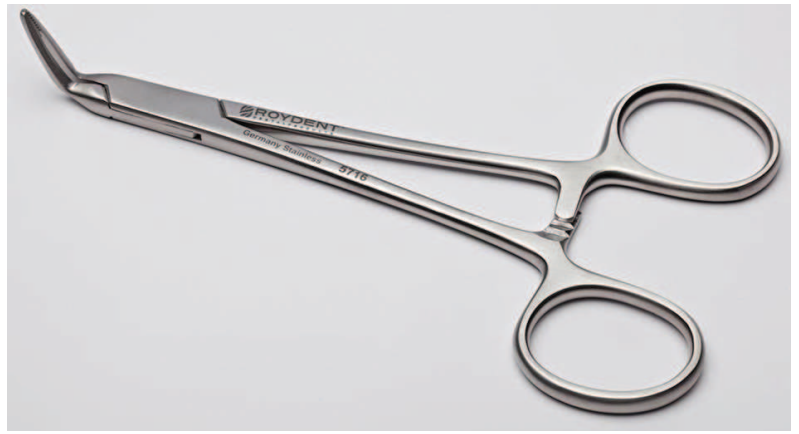

Fig. 1. Endo Forceps (Roydent, Johnson City, TN)

Use of hypodermic surgical needles (Fig. 2) the technique involves a shortened hypodermic needle to create a groove around the coronal part of the fragment. This groove can be created also with ultrasonic tips. Strong dental cement or glue can then be inserted into the needle and the whole complex needle-glue-fragment can be carefully pulled out. In cases when glue or cement cannot be used, an $\mathrm{H}$-file can be applied through the needle and after good interlocking between the fragment and the $\mathrm{H}$-file - both are pulled out $(2,23)$.

A disadvantage of the technique is that it cannot be used in curved root canals. Some authors describe applying the Tube-and-Hedstroem file method as an option in cases when the ultrasound technique does not work (21). The surgical needle is replaced with a microtube. The exact technique in-

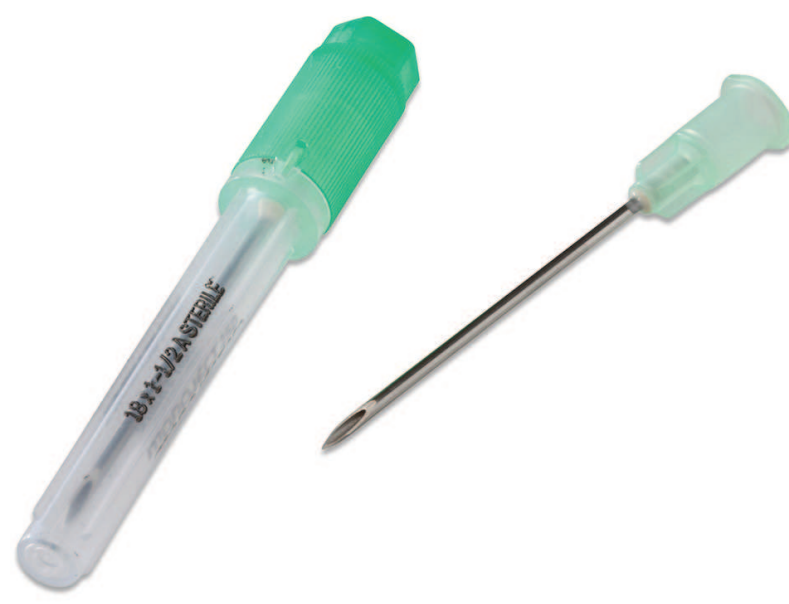

Fig. 2. Hypodermic surgical needle

volves choosing the correct microtube that could reach the exposed coronal part of the fragment. $\mathrm{H}$ file is selected and inserted into the microtube and passed down the length of the tube until tight contact between the fractured segment and the internal lumen of the microtube is achieved (11).

$\diamond$ Using Masserann instruments (Fig. 3): The Masserann kit consists of 14 hollow cutting-end trephine burs, ranging in diameter, and two extractors. The burs are used in counterclockwise direction to create a groove around the coronal part of the fragment. The large diameter of the extractors requires removal of a large amount of dentin, which results in weakening of the tooth and may lead to iatrogenic perforations and fractures (2). The extractors are suggested for use in anterior teeth and straight root canals (1).

$\diamond$ Extractors, for example the Cancellier Extractor Kit (Sybron Endo, Orange, CA), have been developed as a modification of the Masserann

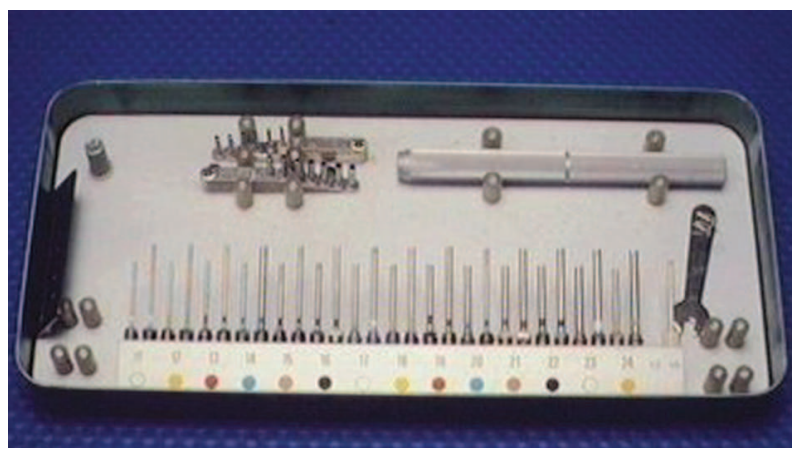

Fig. 3. Masserann instrument kit 
instruments kit. Each extractor has its corresponding bur that creates the platform around the coronal part of the segment (2). Restrictions exist for the use of the burs - they should only be used in a straight part of the root canal. When adhesives are used, extractors can be very effective, especially in cases when the fragment is already loosened. However, too much adhesive can lead to blockage of the RC, and the excessive dentin removal - to perforations and weakening of the tooth (24).

$\diamond$ Ultrasound technique: Ultrasound is used in endodontics not only for gaining access, cleaning and shaping of the root canal, removal of intracanal materials and apical surgery, but also for the management of separated instruments (25).

The successful removal requires access to the separated fragment, which can be created with hand files. After shaping the canal, ultrasonic technique can be used to remove the broken instrument. In order to assure enough space lateral of segment a modified Gates Glidden (GG) (Fig. 4) is used to create a circumferential platform around its coronal part. This procedure is followed by ultrasonic instrumentation - the tip of the selected instrument (Fig. 5) is placed in the obstruction zone and activated within the lower power settings which results in sanding away den-

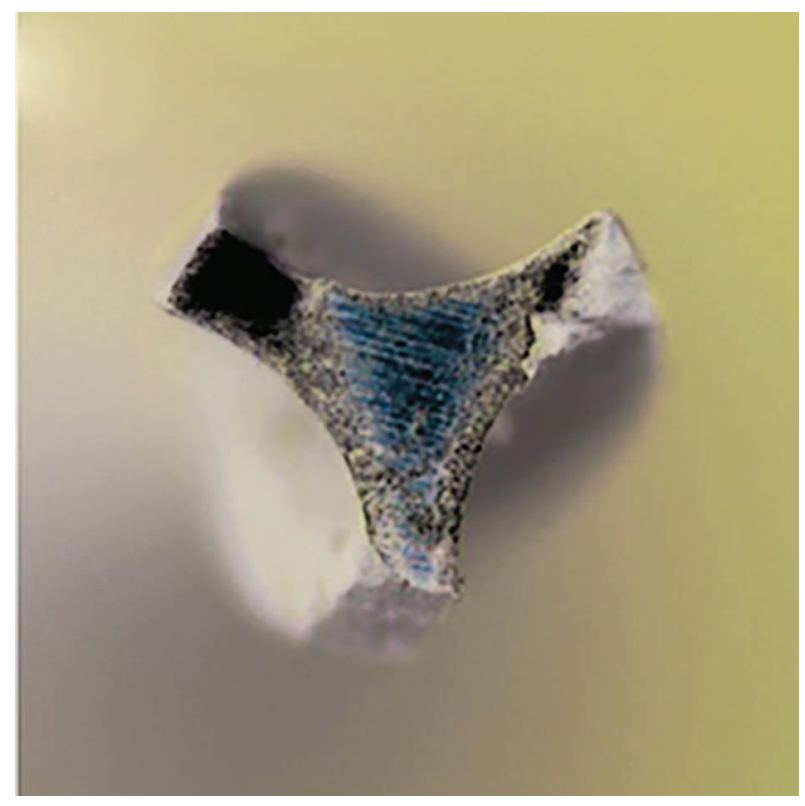

Fig. 4. Modified Gates Glidden (GG) tin and loosening of the instrument. The point of an ordinary GG is modified by cutting it perpendicular at its maximum diameter (Fig. 6). As the right GG for this procedure is considered the one, whose maximum diameter is slightly larger than the diameter of the separated instrument $(1-3,11,25)$.

The technique with modified GG and ultrasound tips and files is the most commonly reported technique, used for the removal of separated instruments $(3,12)$.

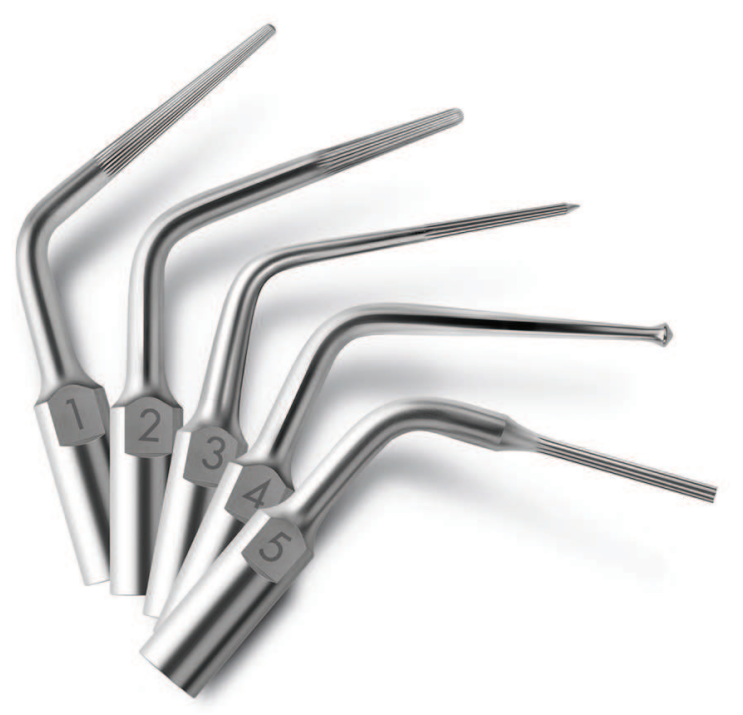

Fig. 5. Ultrasonic tips

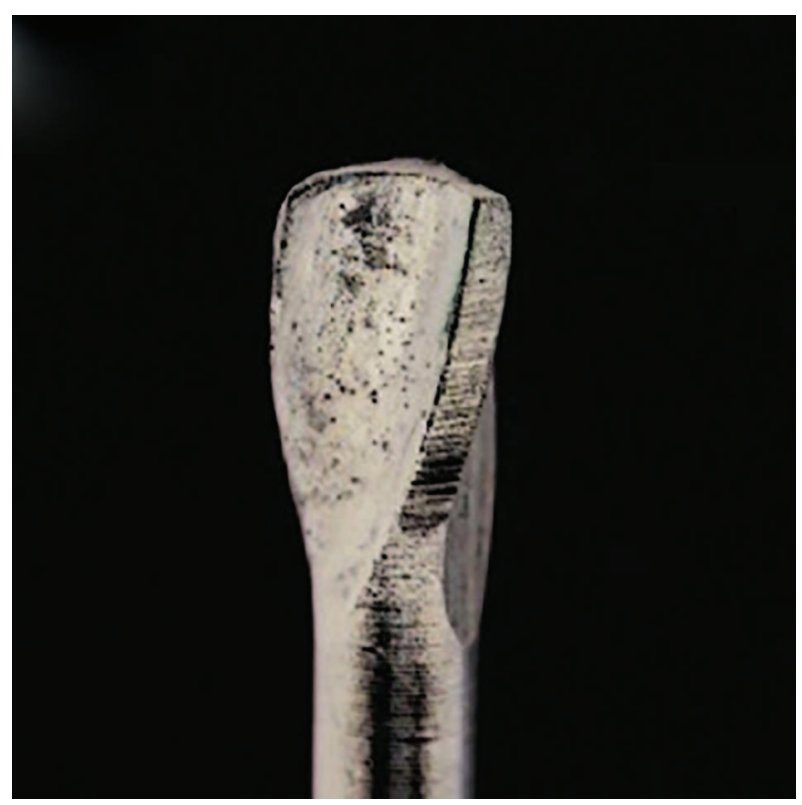

Fig. 6. Modified Gates Glidden (GG) 
$\diamond$ File removal system - this system was developed by Teranchi et al. and consists of 3 steps and is claimed to lead to removal of the fragment with minimum dentin loss.

Step 1 - usage of two low-speed burs - cutting burs A and B;

Step 2 - an ultrasonic tip is used to create a platform around the fragment;

Step 3 - a file removal device is used to mechanically engage and pull the fragment out $(2,26,27)$.

Bypassing describes a procedure, in which a file is inserted between the separated instrument and the root canal dentin until achieving full working length. The fragment remains "in situ" and is later incorporated into the root canal obturation material. This method is considered favorable in many clinical cases because it is more conservative and requires less excessive dentin removal $(20,28,29)$.

Suggesting the bypassing procedure is based on the fact that none of the root canals are perfectly round, so there is always a small gap between the root canal wall and the fragment, which allows a smaller file to bypass the separated fragment. Leaving the fragment in situ and bypassing it with a hand file along with filling the $\mathrm{RC}$ with thermo plasticized gutta-percha improves significantly the prognosis of the tooth (5).

\section{Indications:}

$\diamond$ For fragments located in the middle or apical $1 / 3$ of the RC $(2,5)$;

$\diamond$ For fragments beyond the curvature $(2,5)$;

$\diamond$ After failure of a previous removal attempt;

$\diamond$ In cases when perfect visualization and straightening of the RC cannot be achieved or would be too structurally invasive $(5,11,22)$.

\section{Advantages:}

$\diamond$ More conservative procedure than the complete removal of the fragment $(20,28,29)$;

$\diamond$ Less invasive (18);

$\diamond$ Relatively simple procedure $(5,18)$;

$\diamond$ Often results in complete removal of the fragment $(5,13,21,29)$;

$\diamond$ Allows achieving full working length, proper preparation, irrigation and obturation of the RCS.
Risks:

$\diamond$ Possibility to create a false RC, parallel to the original one $(2,21)$;

$\diamond$ Ledge formation (2);

$\diamond$ Possible perforations $(6,21)$;

$\diamond$ Possible secondary separation of instruments (2);

$\diamond$ Possible fragment extrusion trough the apical foramen $(2,6,18)$.

In many cases the bypassing procedure can be considered an initial step towards removal, because often the initial bypassing results in complete loosening of the fragment $(2,5,13,21)$.

Techniques for bypassing an intracanal separated instrument:

Canal Finder System: This is a system that assists the bypassing of the fragment. The original system (FaSociete Endo Technique, France) consisted of a handpiece and specially designed files. The system produces a vertical movement with amplitude up to 1-2 mm, which decreases when the speed increases. The original system has been replaced later by EndoPulse System (Endo Technic, CA) (Fig.7), which consists of files, used in vertical reciprocation (2).

Ultrasound technique: The successful bypassing starts with creating access to the separated segment. Shaping of the RC above the obstruction is fol-

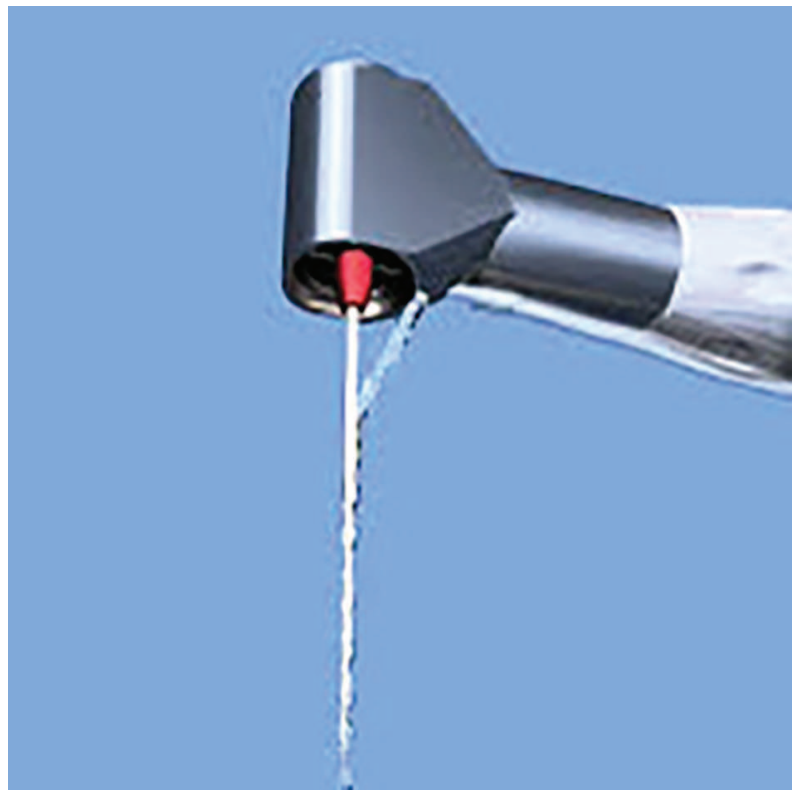

Fig. 7. EndoPulse System (Endo Technic, CA) 
Tsvetelina Borisova-Papancheva, Silvia Stankova, Slavena Georgieva

lowed by evaluation of the broken instrument diameter and the one of the root canal - in cases when the space lateral to the fragment is not enough to place an ultrasonic tip or a handfile a circumferential platform around the coronal part of the instrument can be created using modified GG. This can be followed by creating space at one side of the fragment using ultrasonic tips. The final bypassing is performed with handfiles (K-files ISO 8/10) until achieving full working length. Ultrasonic files (Fig. 8) can be used to create a thin space between the dentin of the $\mathrm{RC}$ and the fractured instrument in order to make the bypassing procedure possible.

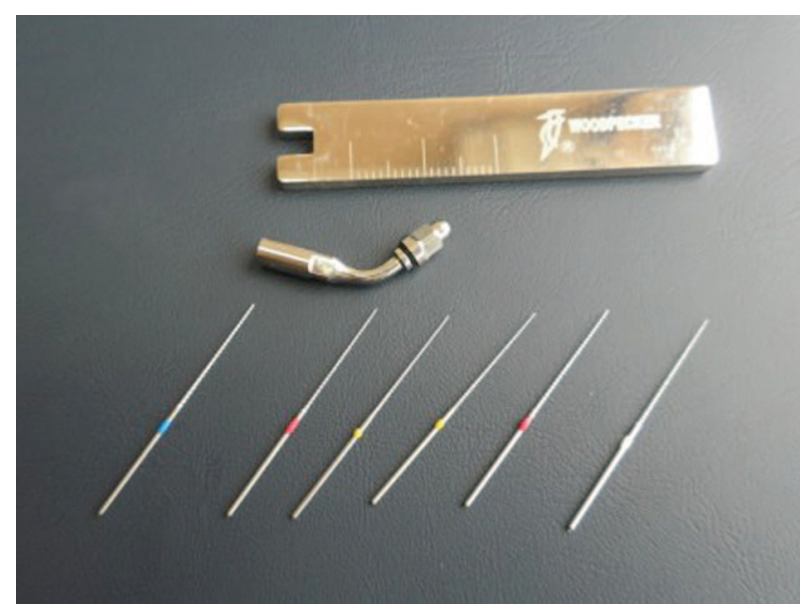

Fig. 8. Ultrasonic files

The bypassing method and the complete removal of a separated endodontic instrument both require good visualization. Studies prove two times greater success of the management when the fragment is visible inside the RC (30). The use of operating microscope, which assures direct and illuminated visualization of the coronal part of the instrument, is considered essential (3). Different studies prove higher success rate in removing the fragment with the use of a microscope $(13,21)$.

\section{DISCUSSION}

Procedural errors in endodontics can happen during every endodontic treatment. They can be the result of factors over which the operator may or may not have control (9).

Management of separated instruments is a complex problem that should be solved in every clinical situation according to the specific related factors.
The fractured instrument itself is not a reason for endodontic failure, but the results of the separation, such as blockage of the $\mathrm{RC}$ and creating an obstacle for the proper instrumentation, irrigation and obturation may compromise the long-term prognosis of the tooth involved.

Separated instruments can be handled conservatively or surgically. The conservative methods such as leaving the fragment in situ and filling the RC to the coronal level of the instrument, bypassing the segment or removing it from the $\mathrm{RC}$ are considered favorable and preferred whenever possible.

The main determinant for removal of the separated instrument is the location of the fragment in the RC and its relation to the root curvature (9). Complete removal of the fragments is considered possible when $1 / 3$ or more of the fragment is exposed (11), in cases when good visualization is achievable $(11,22)$ for fragments located before the root canal curvature, in the coronal $1 / 3$ of the RC $(11,19,22)$ and in straight root canals (11). Removal of the separated instrument is accompanied by risks of iatrogenic events and disadvantages, such as possible ledge formation, stripping perforations, possible extrusion of the separated fragment apically or through the apical foramen (19), requires additional preparation and excessive dentin loss $(3,22)$ and therefore can result in weakening the root structure and vertical root fractures $(19,22)$.

The bypassing procedure is considered more conservative and less structurally invasive because it does not require such excessive dentin removal from the root canal wall. It is a relatively simple procedure $(5,18)$ that often results in complete removal of the fragment $(5,13,21,29)$ and allows the achievement of full working length, proper preparation, irrigation and obturation of the root canal system. It also presents the same risks of iatrogenic events like the removal procedures in addition to the possibility to create a false RC, parallel to the original one $(2,21)$.

Leaving the fragment in situ is considered an adequate treatment option only in cases without periapical pathology and for fragments located in the apical $1 / 3$ of the RC $(2,5)$.

After careful evaluation of the clinical situation, prognosis and restorative status of the tooth the operator should make the management decision - to re- 
move or to leave the fragment in the root canal, while keeping in mind that the successful treatment should not be limited to simply removing the fragment, but should focus more on preserving the long-term integrity and longevity of the tooth.

\section{CONCLUSION}

Instrument separation can happen even if the instrumentation protocol was followed precisely (22). The decision for conservative management to bypass or to completely remove the fractured instrument should be made after careful evaluation of various factors, such as root canal system anatomy and morphology, exact location of the fragment, presence or absence of periapical pathology and armamentarium available. No standardized procedure for the successful management of separated instruments exists. The clinician should consider all the related to the specific clinical situation factors in order to make a balanced decision - possible success against potential risks

\section{REFERENCES}

1. Ruddle CJ. Nonsurgical Retreatment. J Endod. 2004; 30(12): 827-45. doi: 10.1097/01. don.0000145033.15701.2d

2. Madarati AA, Hunter MJ, Dummer PM. Management of Intracanal Separated Instruments. J Endod. 2013; 39(5):569-81. doi: 10.1016/j. joen.2012.12.033

3. McGuigan MB, Louca C, Duncan HF. Clinical decision-making after endodontic instrument fracture. Br Dent J. 2013; 214 (8):395-400. doi: 10.1038/ sj.bdj.2013.379

4. Patel JJ, Morawala A, Talathi R, Shirol D. Retrieval of a broken instrument from root canal in primary anterior teeth. Univ Res J Dent. 2015; 5 (3): 203-6. doi: 10.4103/2249-9725.162799

5. Rambabu T. Management of fractured endodontic instruments in root canal: a review. J Sci Dent. 2014; 4(2):40 - 48 .

6. Cohen SJ, Glassman GD, Mounce R. Rips, Strips and Broken Tips: Handling the Endodontic Mishap Part I: The Separated Instrument. Oral Health. 2005: 10-20.

7. Patiño PV, Biedma BM, Liébana CR, Cantatore G, Bahillo JG. The Influence of a Manual Glide Path on the Separation Rate of NiTi Rotary Instru- ments. J Endod. 2005; 31(2): 114-6. doi: 10.1097/01. don.0000136209.28647.13

8. Bahcall JK, Carp S, Miner M, Skidmore L. The causes, prevention, and clinical management of broken endodontic rotary files. Dent Today. 2005; 24 (11): 74, 76, 78-80.

9. Choksi D, Idnani B, Kalaria D, Patel RN. Management of an Intracanal Separated Instrument: A Case Report. Iran Endod J. 2013;8(4):205-7.

10. Patel B. Endodontic Treatment, Retreatment and Surgery, Mastering Clinical Practice. Springer 2016.

11. Ruddle CJ. Broken Instrument Removal: The Endodontic Challenge. Dent Today. 2002; 21(7):70-2, 74,76 .

12. Gencoglu N, Helvacioglu D. Comparison of the Different Techniques to Remove Fractured Endodontic Instruments from Root Canal Systems. Eur J Dent. 2009; 3(2): 90-95.

13. Cujé J, Bargholz C, Hülsmann M. The outcome of retained instrument removal in a specialist practice. Int Endod J. 2010;43(7):545-54. doi: 10.1111/j.1365-2591.2009.01652.x

14. Gencoglu N, Helvacioglu D. Comparison of the different techniques to remove fractured endodontic instruments from root canal systems. Eur J Dent. 2009;3(2):90-5.

15. Madarati AA, Hunter MJ, Dummer PM. Management of intracanal separated instruments. J Endod. 2013;39(5):569-81. doi: 10.1016/j.joen.2012.12.033

16. Pedir SS, Mahran AH, Beshr K, Baroudi K. Evaluation of the Factors and Treatment Options of Separated Endodontic Files Among Dentists and Undergraduate Students in Riyadh Area. J Clin Diagn Res. 2016; 10(3): 18-23. doi: 10.7860/ JCDR/2016/16785.7353

17. Hülsmann $\mathrm{M}$, Schinkel I. Influence of several factors on the success or failure of removal of fractured instruments from the root canal. Endod Dent Traumatol. 1999; 15(6):252-8. doi: 10.1111/j.16009657.1999.tb00783.x

18. Parveen S, Hossain M, Uddin F. Management of broken instrument by file bypass technique. BSMMU J. 2017; 10(1): 41-43. doi: 10.3329/bsmmuj. v10i1.31305

19. Souter NJ, Messer HH. Complications associated with fractured file removal using an ultrasonic technique; J Endod. 2005; 31(6):450-2. doi: 10.1097/01.don.0000148148.98255.15 
20. Shen Y, Peng B, Cheung GS. Factors associated with the removal of fractured NiTi instruments from root canal systems. Oral Surg Oral Med Oral Pathol Oral Radiol Endod. 2004; 98(5):605-10. doi: $10.1016 / S 1079210404002884$

21. Suter B, Lussi A, Sequeira P. Probability of removing fractured instruments from root canals. Int Endod J. 2005;38(2):112-23. doi: 10.1111/j.1365-2591.2004.00916.x

22. Praveen J, Kumar R, Jayasree S. Bypassing separated instruments in the root canal - two case reports. IOSR Journal of Dental and Medical Sciences. 2016; 15 (6): 8-13. doi: 10.9790/0853-1506110613

23. Frota LM, Aguiar BA, Aragão MG, de Vasconcelos BC. Removal of Separated Endodontic K-File with the Aid of Hypodermic Needle and Cyanoacrylate. Case Rep Dent. 2016. doi: 10.1155/2016/3970743

24. Yoldas O, Oztunc H, Tinaz C, Alparslan N. Perforation risks associated with the use of Masserann endodontic kit drills in mandibular molars. Oral Surg Oral Med Oral Pathol Oral Radiol Endod. 2004; 97(4):513-7. doi: 10.1016/S1079210403005675

25. Plotino G, Pameijer CH, Grande NM, Somma F. Ultrasonics in endodontics: a review of the literature. J Endod. 2007; 33 (2): 81-95. doi: 10.1016/j. joen.2006.10.008

26. Terauchi Y, O'Leary L, Kikuchi I, Asanagi M, Yoshioka T, Kobayashi C, et al. Evaluation of the efficiency of a new file removal system in comparison with two conventional systems. J Endod. 2007;33(5):585-8. doi: 10.1016/j.joen.2006.12.018

27. Terauchi Y, O'Leary L, Suda H. Removal of separated files from root canals with a new file-removal system: Case reports. J Endod. 2006;32(8):789-97. doi: 10.1016/j.joen.2005.12.009

28. Al-Fouzan KS. Incidence of rotary ProFile instrument fracture and the potential for bypassing in vivo. Int Endod J. 2003; 36(12):864-7. doi: 10.1111/j.1365-2591.2003.00733.x

29. Jadhav GR. Endodontic management of a two rooted, three canaled mandibular canine with a fractured instrument. J Conserv Dent. 2014; 17(2): 1925. doi: 10.4103/0972-0707.128046

30. Nevares G, Cunha RS, Zuolo ML, Bueno CE. Success rates for removing or bypassing fractured instruments: a prospective clinical study. J Endod. 2012 ; 38(4):442-4. doi: 10.1016/j. joen.2011.12.009 